\title{
A Prova dE TRabalho E a VIA DE PARTO EM PRIMÍPARAS COM UMA CESÁREA ANTERIOR
}

Jacinta Pereira Matias, Mary Angela Parpinelli", José Guilherme Cecatti

Trabalho realizado pela Área de Obstetrícia, Departamento de Tocoginecologia da Faculdade de Ciências Médicas, Universidade Estadual de Campinas (SP).

*Correspondência:

Rua Alexander Fleming, 101 Campinas/SP

Cep: $13081-970$

Tel/fax: (19) 3788-9304

parpinelli@caism.unicamp.br

\section{RESUMO}

OBjetivo. Identificar fatores associados à cesárea em mulheres com um único parto anterior por cesárea e submetidas à prova de trabalho de parto.

Métodos. Estudo de corte transversal retrospectivo, incluindo 1746 mulheres com uma cesárea anterior submetidas à prova de trabalho de parto no segundo parto ocorrendo entre 1986 e 1998. Foram exclúidos os casos com atual gestação múltipla e/ou com malformações fetais incompativeis com a vida. Elas foram divididas pelo tipo de parto atual em dois grupos: cesárea $(n=73 \mid)$ e parto vaginal após cesárea (PVAC, $n=|0| 5)$. A análise estatística para a identificação de fatores associados ao tipo de parto foi feita por meio do cálculo da razão de prevalência (RP) e IC 95\%, sendo feito também o ajuste por idade, excluindose os casos com informações ignoradas em cada análise.

Resultados. A taxa total de parto vaginal após cesárea foi de $58,1 \%$. Os fatores significativamente associados com parto por cesárea foram: maior idade materna, maior altura uterina (RP I,5; IC 95\% I, I9-1,88), rotura prematura de membranas (I,3; I,08-1,54), líquido amniótico não claro $(1,22 ; 1,04-1,43)$ ou com quantidade alterada $(1,32 ; 1,01-1,73)$, alteração dos batimentos cardíacos fetais $(1,96 ; 1,68-2,28)$, apresentaçãa não cefálica $(2,03 ; 1,54-2,66)$, indução do parto $(1,74 ; 1,42$ 2, II) e ausência de analgesia $(2,57 ; 2,11-3$, II).

Conclusão. Os fatores associados ao parto por cesárea foram a maior idade, apresentação não-cefálica, rotura prematura de membranas, fetos grandes, necessidade de induçãa do trabalho de parto, e sinais de comprometimento da vitalidade fetal.

Untermos: Cesárea. Fatores de risco. Prova de trabalho de parto. Parto vaginal após cesárea.

\section{INTRODUÇão}

No decorrer do século XX, observou-se o crescimento linear das taxas de cesárea em todo o mundo devido principalmente à diminuição da morbidade materna associada à sua realização, sobretudo após o advento da antibioticoterapia e das modernas técnicas de hemoterapia. Na década de 60, com o surgimento de novos métodos para avaliação da vitalidade fetal, foram acrescentadas às indicações maternas de cesárea aquelas por sofrimento fetal, crescendo, assim, sua popularidade e frequêência. Paralelamente, alcançou-se, no decorrer deste período, a simplificação da técnica de sua execução e a redução significativa da morbi-mortalidade perinatal!' No entanto, esta redução parece relacionar-se mais diretamente ao desenvolvimento da assistência à gravidez, ao parto e ao recémnascido do que à via de parto propriamente dita'. Algumas evidências neste sentido podem ser encontradas em países desenvolvidos, onde a mortalidade materna e perinatal declinaram, mesmo em vigência de taxas de cesárea baixas e estáveis².

Apesar destas considerações, a aparente correlação entre os bons resultados materno-fetais e a via de parto tem servido de argumento para o aumento vertiginoso da realização de cesáreas ao redor do mundo, principalmente no Brasil, onde seus índices podem ser considerados alarmantes, ainda que não mais ostente, atualmente, o título de "campeão mundial de cesáreas", agora disputado entre Chile e Coréia do Sul3-5.

Embora seja um procedimento de execução relativamente simples, a cesárea apresenta riscos inerentes à sua indicação, sobretudo quando de repetição. O risco de morte materna é maior, bem como a ocorrência de infecção, hemorragia e acidentes anestésicos, que tornam a morbidade materna também maior que no parto vaginal'. Complicações tardias, principalmente a placenta prévia com variados graus de acretismo, estão diretamente relacionadas ao antecedente de cesárea'. Com relação ao feto, embora a cesárea seja salvadora de vidas quando indicada por prolapso de cordão, descolamento prematuro de placenta, desproporção cefalopélvica e sofrimento fetal agudo, por exemplo, também pode levar a maior risco de prematuridade iatrogênica e desconforto respiratório neonatal, quando realizada sem uma indicação precisal,3,6.

Ainda é relativamente comum o argumento de que uma cicatriz uterina prévia justifica a repetição da cesárea devido ao maior risco de rotura uterina ${ }^{7}$. A assertiva de $\mathrm{Cragin}^{8}$ de que "uma vez cesárea, sempre cesárea" deu-se em 1916 quando as histerotomias eram segmento-corporais (longitudinais). Atualmente, contudo, procede-se à histerotomia segmentar transversa na imensa maioria dos casos, o que reduz a ocorrência de complicações.

A repetição da cesárea, no entanto, é um dos principais fatores contribuintes para as altas taxas, ao lado das crescentes pressões médico-legais sobre os obstetras, da realização de monitorização fetal eletrônica por pessoal não treinado adequadamente e da diminuição da experiência em parto vaginal operatório, embora a segurança de uma tentativa de trabalho de parto depois de uma cesariana transversa baixa prévia venha sendo confirmada por inúmeros autores, desde que recebam atenção especializada e de 
MATIAS JP ET AL.

qualidade ${ }^{9}$. Embora exista ainda controvérsia, há uma tendência em se considerar que os benefícios do parto vaginal após cesárea (PVAC) superam os riscos materno-fetais na maioria das mulheres e de que a implementação da prova de trabalho de parto (PTP) constitui estratégia legítima para se diminuir o índice de cesáreas desnecessárias ${ }^{10,11}$, embora atualmente também se reconheça que existe um risco inerente ao procedimento, especialmente se o trabalho de parto for induzido ${ }^{12}$.

Devido ao fato de que, até o momento, não existem estudos randomizados e controlados que demonstrem que os resultados maternos e neonatais são melhores com a prova de trabalho de parto do que com cesárea repetida eletivamente, e também porque as mulheres de fato preferem o parto vaginal mesmo que tenham já tido uma cesárea ${ }^{13}$, é necessário que se busque estabelecer critérios para a identificação das mulheres com maior chance de sucesso numa PTP, bem como daquelas para as quais a PTP acarrete maior risco de morbi-mortalidade, tanto materna quanto perinatal|1-17. De fato, a revisão sistemática da Biblioteca Cochrane disponível sobre o tema também confirma a não existência de estudos controlados e aleatorizados que possam fornecer evidências definitivas sobre qual a melhor alternativa de intervenção nessa situação, conhecendo-se vantagens e desvantagens de ambas as abordagens ${ }^{18}$.

Assim, o objetivo deste estudo foi identificar fatores que se associaram à evolução para cesárea em um grupo de mulheres com um único parto anterior por cesárea e submetidas à PTP.

\section{Métodos}

Trata-se de um estudo retrospectivo de corte transversal. As variáveis foram coletadas do banco de dados institucional que contém as informações sobre todos os partos ocorridos no Centro de Atenção Integral à Saúde da Mulher (CAISM) da Universidade Estadual de Campinas. Este banco de dados existe desde 1977 e é constituído pelas informações de todas as gestantes que deram à luz na instituição, em idades gestacionais acima de 20 semanas ou peso fetal maior ou igual a $500 \mathrm{~g}$, independentemente do tipo de parto, número de fetos ou ainda da vitalidade do feto ou recémnascido. Essas informações são transcritas para uma ficha obstétrica pré-codificada, que dispõe de um manual de preenchimento para padronização dos procedimentos e respostas, a partir do momento da internação da gestante e até a sua alta e a do recém-nascido. Essas fichas são logo então conferidas, completadas e corrigidas por um médico responsável pela qualidade das informações e posteriormente digitadas em um banco de dados especialmente estruturado para essa finalidade. Antes de serem disponibilizadas, essas informações são submetidas a testes de limpeza e consistência, com nova correção das informações se necessária, confrontando com o prontuário clínico do caso. Assim, embora o estudo tenha sido retrospectivo, todos os dados foram prospectivamente e cuidadosamente coletados de forma padronizada.

Foram selecionados todos os casos que preenchiam os critérios de inclusão: mulheres com apenas um parto anterior, por cesárea, que foram submetidas a uma prova de trabalho de parto espontânea ou induzida no segundo parto. Do total de 32.400 partos ocorridos na instituição no período entre 1986 e 1998, 2109 (6,5\%) eram de mulheres que tinham tido uma única cesárea anterior. Foram excluídos os casos em que a segunda gestação era múltipla (25 casos, porque essa é uma indicação de cesárea eletiva no serviço) e/ou com recém-nascido portador de malformações incompatíveis com a vida ( 16 casos, porque a vitalidade neonatal associada com a prova de trabalho de parto também foi estudada ${ }^{15}$ ), restando 2068 mulheres. Destas, 322 tiveram indicada uma cesárea eletiva, sem prova de trabalho de parto.

Durante todo o período, como norma do serviço, às mulheres com uma cesárea anterior e sem indicação médica de repetição da cesárea foi permitido que entrassem em trabalho de parto espontâneo. Naquelas em que isso não aconteceu e que apresentaram alguma indicação de interrupção da gestação, o trabalho de parto foi induzido com ocitocina (I74 casos). De acordo com a padronização do serviço, a indução com ocitocina das mulheres com uma cicatriz de cesárea é realizada com uma infusão endovenosa de ocitocina administrada por bomba de infusão, preparada com a adição de $5 \mathrm{Ul}$ de ocitocina em $500 \mathrm{ml}$ de soro glicosado a $5 \%$, resultando em uma concentração de ocitocina de $10 \mathrm{mU} / \mathrm{ml}$, iniciando com uma dose de $6 \mathrm{um} / \mathrm{min}$ e aumentando o gotejamento em 3mU/min em intervalos de 30-60 min até um máximo de 42 $\mathrm{mU} / \mathrm{min}$ ou trabalho de parto estabelecido. Isso correspondeu à prova de trabalho de parto que foi realizada em 1.746 mulheres que constituem a amostra deste estudo.

As mulheres participantes do estudo foram divididas, de acordo com o tipo de parto da gestação atual como evolução da prova de trabalho de parto $(n=1746)$, em dois grupos para análise: cesárea $(n=731)$ e PVAC $(n=1015)$. As variáveis estudadas foram: idade materna, estado marital, escolaridade, altura da mulher, intervalo interpartal, vitalidade do primeiro filho (filho vivo, morto ou natimorto), presença de antecedentes mórbidos (clínico ou obstétrico), aborto anterior, número de consultas de pré-natal, altura uterina (AU), ocorrência de rotura prematura de membranas (RPM), estado e quantidade do líquido amniótico (LA) no momento do parto, alteração dos batimentos cardíacos fetais (BCF) à ausculta clínica ou pela cardiotocografia (desacelerações tardias e/ou bradicardias sustentadas), apresentação fetal, tempo de rotura de membranas até o parto (TRM-P), indução do TP, realização de analgesia de parto (analgesia peridural) e indicação da cesárea para os casos que tiveram esse tipo de parto.

Inicialmente são apresentadas as variações da prevalência da evolução da PTP para cesárea ou PVAC em função do tempo. A análise estatística para a identificação de fatores associados à forma de término do parto após PTP foi feita pelo cálculo da razão de prevalência (RP) e IC 95\%, sendo feito também o ajuste por idade para cada variável por regressão linear simples. Este estudo foi aprovado pelo Comitê de Ética em Pesquisa da Faculdade de Ciências Médicas da Unicamp.

\section{Resultados}

O total de casos identificados no período de 13 anos do estudo foi de 1746, sendo 731 cesáreas após PTP e 1015 PVAC, o que corresponde a uma taxa total de PVAC de 58,1\%. No decorrer do tempo estudado, a evolução da PTP para cesárea diminuiu gradativamente, havendo um correspondente aumento na evolução para PVAC neste grupo de mulheres, com um aumento total de cerca de 20\% em 13 anos, chegando a cerca de $70 \%$ em 1998. 
$\mathrm{Na}$ Tabela I, observa-se que, dentre os aspectos sociodemográficos estudados, apenas a idade esteve associada à evolução para cesárea após PTP. O grupo de mulheres de 35 anos ou mais apresentou quase 2,5 vezes mais partos por cesárea. Quanto aos antecedentes obstétricos, tanto aborto quanto condição patológica obstétrica anterior estiveram inicialmente associados ao término do parto por cesárea após PTP, porém estas associações desapareceram após o ajuste por idade. A Tabela 2 mostra as principais indicações de cesárea para as mulheres que tiveram uma prova de trabalho de parto não exitosa, chamando a atenção o número proporcionalmente menor de indicações por hipertensão arterial e gestação prolongada do que nas cesáreas eletivamente indicadas.

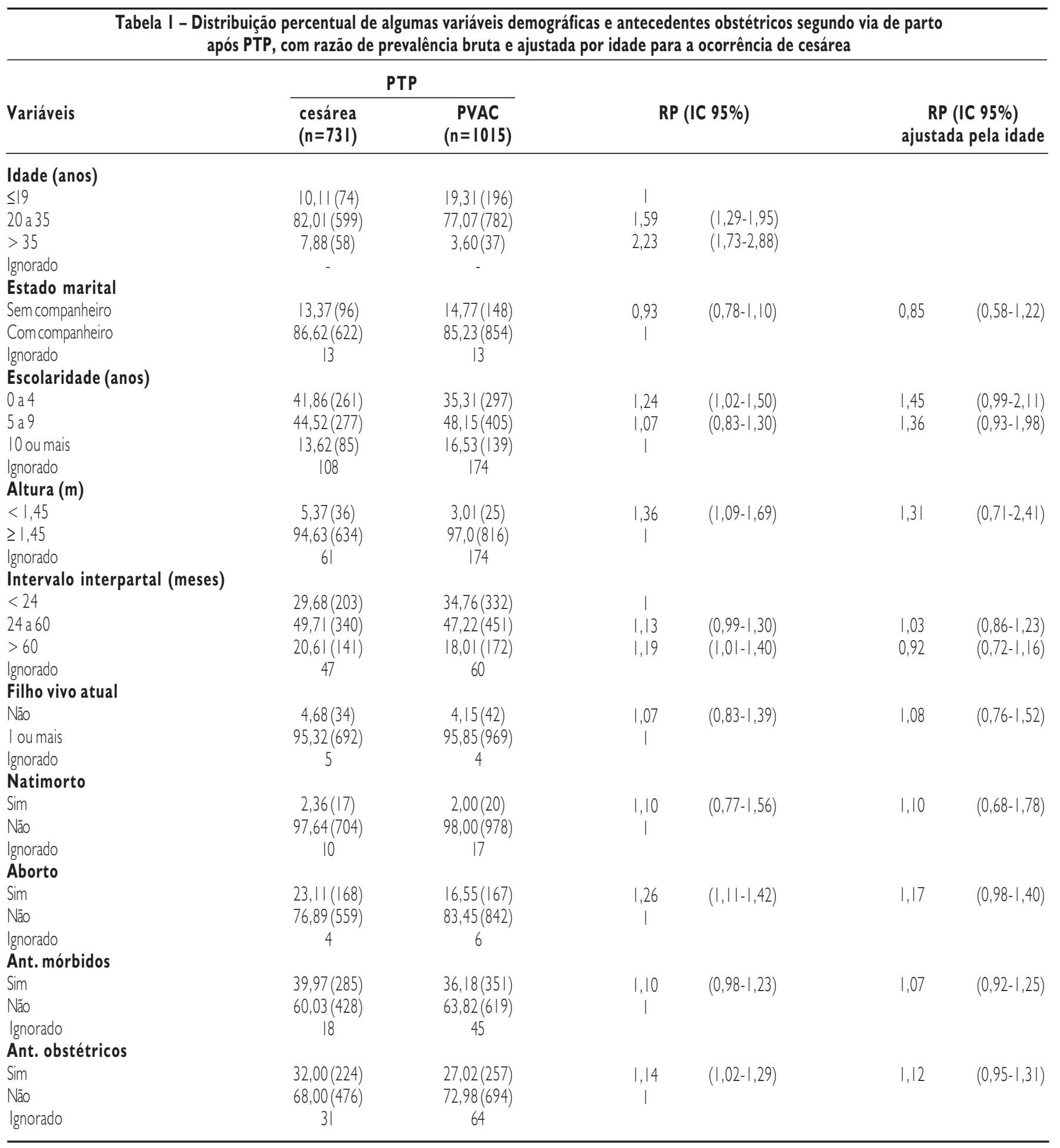


MATIAS JP ET AL.

\begin{tabular}{|c|c|c|}
\hline Indicação da cesárea & $\mathrm{n}$ & $\%$ \\
\hline $\begin{array}{l}\text { Sofrimento fetal } \\
\text { Desproporção céfalo-pélvica } \\
\text { Distócias } \\
\text { Falha de indução } \\
\text { Síndrome hipertensiva } \\
\text { Gestação prolongada } \\
\text { Outras } \\
\text { Total }\end{array}$ & $\begin{array}{l}206 \\
165 \\
95 \\
41 \\
23 \\
22 \\
179 \\
731\end{array}$ & $\begin{array}{r}28,4 \\
22,8 \\
12,9 \\
5,7 \\
3,2 \\
3,0 \\
24,5 \\
100,0\end{array}$ \\
\hline
\end{tabular}

Ao serem analisadas as variáveis relativas à gestação atual (Tabela 3), observou-se que a RPM, a apresentação fetal não cefálica e AU maior que $39 \mathrm{~cm}$ associaram-se ao término da PTP por cesárea. No que diz respeito às possíveis alterações da vitalidade fetal avaliadas pela presença de LA não claro ou em volume alterado, bem como pelas alterações dos BCF, também houve associação com o término da PTP por cesárea, mesmo após o ajuste pela idade. Quanto às variáveis do trabalho de parto em questão, a Tabela 3 mostra, ainda, que a indução do TP aumentou em $70 \%$ o risco para cesárea, que também foi 2,5 vezes maior quando não se realizou analgesia de parto.

\section{Discussão}

Os resultados observados mostraram que a evolução da PTP para cesárea diminuiu ao longo do período estudado, havendo um correspondente aumento da evolução para PVAC, e que existem alguns fatores que se associam à evolução para cesárea. Nos anos iniciais do estudo, o percentual de cesáreas após PTP girava em torno de 50\%, com pequeno predomínio da evolução para PVAC, até 1993. A partir daí, observa-se uma expressiva tendência para o PVAC, superando a cifra de 70\%, em 1994, e mantendo-se neste patamar nos dois últimos anos do estudo.

Este panorama parece refletir as observações feitas em nosso meio, no sentido de se implementar a PTP em mulheres com o antecedente de uma cesárea como uma das estratégias para se diminuir a exorbitante taxa nacional deste procedimento, uma vez que, nesta época, o Brasil ainda apresentava as maiores taxas conhecidas de cesárea em todo o mundo 14, 17, 19

No entanto, é essencial que este grupo de mulheres seja continuamente estudado a fim de se identificarem aquelas que são candidatas a uma PTP com maior chance de sucesso ${ }^{11,20,21}$, ainda que a literatura médica esteja farta de artigos que demonstrem não haver aumento significativo do risco fetal22. Embora sem contra-indicar a PTP ou o parto vaginal nestas situações, é conhecido o maior risco de rotura uterina devido às contrações em um útero com cicatriz prévia, especialmente se o trabalho de parto for induzido, e principalmente se prostaglandinas forem utilizadas para esta finalidade ${ }^{9,12,23}$, o que não aconteceu com as mulheres do presente estudo.

Algumas características associam-se claramente a uma evolução favorável da PTP para PVAC, tais como a indicação da primeira cesárea por fator não recorrente ${ }^{11,17,24}$ e o antecedente de parto vaginal, no caso das multíparas com uma cesárea anterior". No presente estudo, na análise de algumas características sociodemográficas, observou-se que apenas a idade de 35 anos ou mais esteve nitidamente associada à evolução da PTP para cesárea, como já encontrado em vários outros estudos brasileiros ${ }^{14,17,25}$. Por sua vez, a forte associação da idade menor que 19 anos ao PVAC determinou que se fizesse a opção pelo cálculo da RP também se ajustando pela idade. Dessa forma, aparentes associações desapareceram, tais como aquela entre a evolução da PTP para cesárea e a presença de antecedentes de patologia obstétrica ou de aborto.

Ao se analisarem as características inerentes à gestação em estudo, observou-se que a ocorrência de RPM, apresentação fetal não cefálica e maior altura uterina associaram-se à cesárea após PTP, mesmo após o ajuste por idade. Quanto à RPM nestes casos estar mais associada à cesárea, deve-se considerar a existência de poucos recursos para se melhorar o índice de Bishop visando uma posterior indução com ocitocina, visto que o misoprostol e a sonda de Foley estão contraindicados como agentes maturadores de colo nessa situação. Também era avaliado o risco de infecção ovular após períodos prolongados de amniorrexe e, portanto, em casos em que se previa um longo período entre o momento da rotura da bolsa amniótica e o parto, é possível que se optasse pela realização de cesárea em grande parte dos casos, o que poderia explicar a associação encontrada.

Outro estudo brasileiro, avaliando dados populacionais, encontrou justamente o contrário: uma maior probabilidade de parto vaginal após a rotura prematura de membranas em mulheres com uma cesárea anterior ${ }^{17}$, provavelmente porque esta situação deva corresponder à condição pouco freqüente de uma prova de trabalho de parto real e espontânea. Quanto à apresentação fetal não cefálica, na maioria dos casos corresponde à apresentação pélvica, a associação é consistente com os dados da literatura, permanecendo como condição formal para repetição de cesárea em primíparas com cesárea anterior ${ }^{11,17}$.

Observou-se também que as alterações do LA, tanto no que diz respeito ao seu aspecto quanto à sua quantidade, estiveram associadas ao término da PTP por cesárea. O mesmo foi verificado quanto às alterações dos batimentos cardíacos fetais. Parece claro que a cesárea foi indicada, nestas situações, com o intuito de proteger o feto da anóxia neonatal grave. A associação destas variáveis com a realização de cesárea permaneceu mesmo após o ajuste pela idade e esses achados são consistentes com os conceitos de vigilância da vitalidade fetal durante o trabalho de parto e a indicação de cesárea por sofrimento fetal. Tais resultados, contudo, não têm evidentemente grande importância como fatores preditores para o sucesso de uma prova de trabalho de parto em mulheres com uma cesárea anterior, visto que só poderão ser rastreados em mulheres já em trabalho de parto. Provavelmente esse é o motivo pelo qual estas variáveis não são consideradas nos demais estudos publicados sobre o assunto.

Dentre as características inerentes ao TP propriamente dito, houve uma clara associação da indução do TP, bem como da não realização de analgesia peridural, com a evolução para cesárea, aumentando este risco em 2,5 vezes em ambos os casos e persistindo esta observação mesmo após o ajuste por idade. Estes achados são concordantes com os de outros autores, principalmente no que concerne ao uso de ocitocina. Ainda que a indução 
com ocitocina pareça, por si só, aumentar o risco de rotura uterina ${ }^{12,23,26,27}$, no presente estudo, o uso da ocitocina para condução do TP favoreceu a evolução da PTP para PVAC.

Em relação à analgesia de parto, embora haja evidências de que seu emprego não aumente os índices de cesárea ou de parto operatório, recentemente observou-se que recém-nascidos de mães com cesárea prévia submetidas a PTP sob analgesia peridural foram mais freqüentemente submetidos a testes diagnósticos e intervenções terapêuticas quando comparados aos de mães submetidas a cesárea eletiva ou PTP sem analgesia ${ }^{28}$.

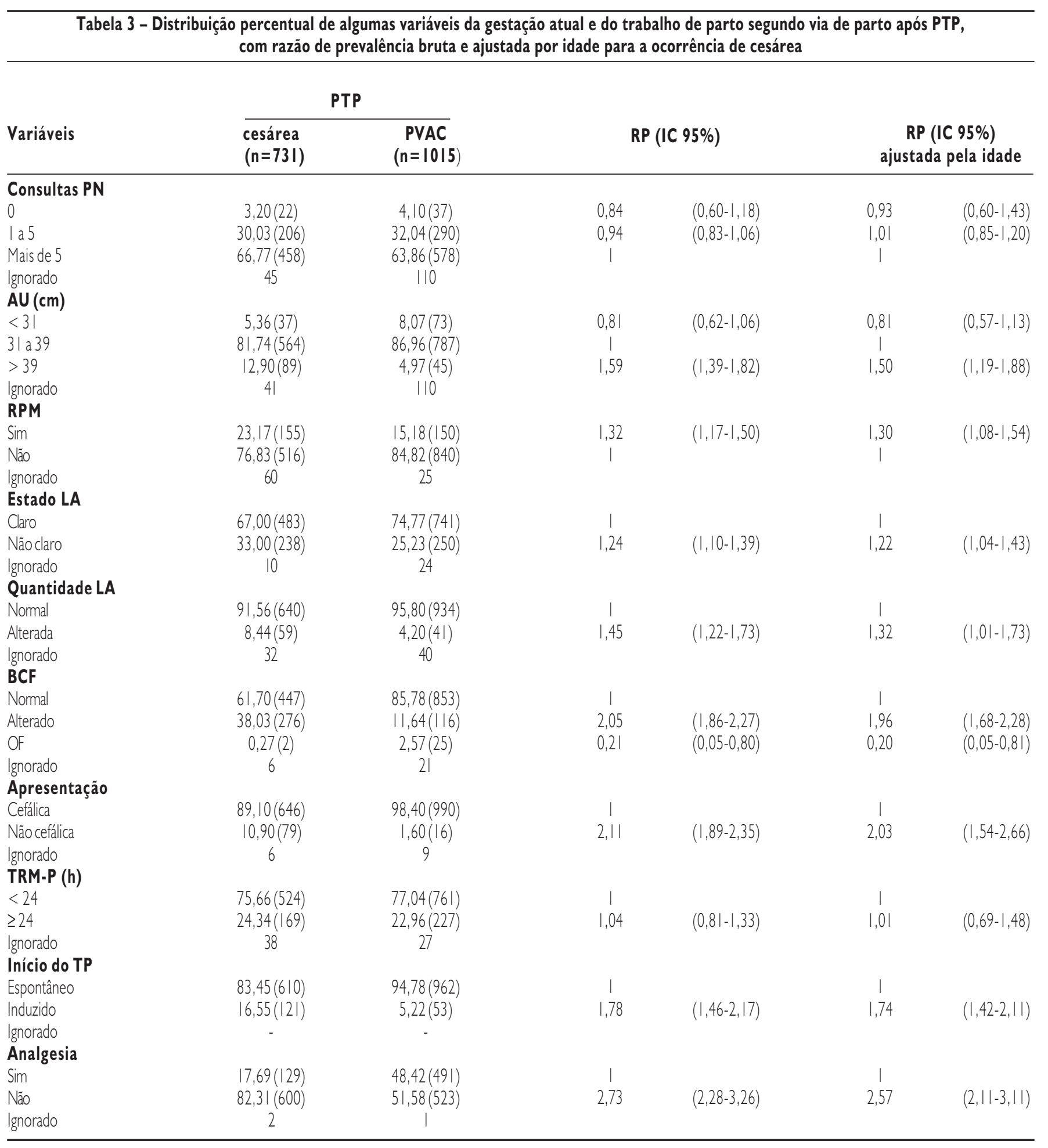


MATIAS JP ET AL.

\section{Discussão}

Dessa forma, pode-se dizer que ainda persistem dúvidas quanto à efetividade e segurança da prova de trabalho de parto para mulheres com uma cesárea anterior. Os dados de uma metanálise sobre o assunto podem resumir a situação do conhecimento atual, mostrando algumas desvantagens para a prova de trabalho de parto (maior risco de rotura uterina, de morte neonatal e de índice de Apgar baixo) e para a cesárea eletiva (maior risco de morbidade febril, de transfusão e de histerectomia) $)^{29}$. Enquanto os dados não forem conclusivos sobre a melhor opção para esses casos (se algum dia de fato isso puder ser conhecido), deverá ser de ajuda conhecer os fatores prognósticos associados ao fracasso da prova de trabalho de parto, numa tentativa de melhor selecionar as mulheres que deveriam ser efetivamente submetidas a este procedimento. De acordo com os resultados do presente estudo, estas gestantes deveriam ser mais jovens, com fetos em apresentação cefálica, sem rotura prematura de membranas, sem sinais de fetos grandes, que entrassem espontaneamente em trabalho de parto, e que não tivessem sinais de comprometimento da vitalidade fetal.

\section{Conflito de interesse: não há.}

\section{SUMMARY}

FACTORS ASSOCIATED WITH MODE OF DELIVERY AMONG PRIMIPARA WOMEN WITH ONE PREVIOUS CESAREAN SECTION AND UNDERGOING A TRIAL OF LABOR

OBJECTIVE. To identify factors associated with cesarean section in women with only one previous delivery by cesarean section and undergoing a trial of labor.

METHODS. A retrospective cross sectional study was performed from 1986 to 1998 including a total of 1746 women with one prior cesarean section and delivering after a trial of labor. Cases with a current twin pregnancy or with malformation incompatible with life were excluded. Women were divided in two groups according to the mode of the second delivery: Cesarean $(n=731)$ or Vaginal Birth After Cesarean (VBAC, $n=1015$ ). To identify factors associated to the mode of delivery, the prevalence ratios (PR) and the $95 \% \mathrm{Cl}$ for each isolated factor, adjusted according to age, were assessed. In each analysis, cases with missing information were excluded.

RESULTS. The total rate of Vaginal Birth After Cesarean was 58.1\%. The factors significantly associated with cesarean section were: higher maternal age, greater fundal height (PR 1.5; 95\% Cl 1.19-1.88), premature rupture of membranes (1.3; 1.08-1.54), amniotic fluid with an altered aspect (1.22; 1.04-1.43) or volume (1.32; 1.01-1.73), altered fetal heart rate (1.96; 1.68-2.28), non cephalic presentation (2.03; 1.54-2.66), induction of labor (1.74; 1.42-2.1/) and no labor analgesia (2.57; 2.1/-3./1).

CONCLUSION. The factors associated with cesarean section were older age, non-cephalic presentation, premature rupture of membranes, signs of large fetus, the need of induction of labor and signs of compromised fetal wellbeing. [Rev Assoc Med Bras 2007; 53(2): 109- I5]
KEY WORDS: Cesarean section. Risk factors. Trial of labor. Vaginal birth after cesarean.

\section{RefERÊNCIAS}

I. Villar J, Valladares E, Wojdyla D, Zavaleta N, Carroli G, Velazco A, et al. Caesarean delivery rates and pregnancy outcomes: the $2005 \mathrm{WHO}$ global survey on maternal and perinatal health in Latin América. Lancet. 2006;367(9525): 1819-29.

2. O'Driscoll K, Foley M. Correlation of decrease in perinatal mortality and increase in cesarean section rates. Obstet Gynecol. 1983;61:1-5.

3. Belizan JM, Althabe F, Barros FC, Alexander S. Rates and implications of cesarean sections in Latin America: ecological study. BMJ. 1999;319:1397-400.

4. Murray SF. Relation between private health insurance and high rates of caesarean section in Chile: qualitative and quantitative study. BMJ. 2000;32I: I 50I-5.

5. Kim C, Ko SK, Kim KY. Are league tables controlling epidemic of caesarean sections in South Korea? Br J Obstet Gynecol. 2005; I I2: $607-11$.

6. Grobman WA, Peaceman AM, Socol ML. Cost-effectiveness of elective cesarean delivery after one prior low transverse cesarean. Obstet Gynecol. 2000;95:745-5I.

7. Flamm BL. Once a cesarean, always a controversy. Obstet Gynecol. 1997;90:312-5.

8. Cragin E. Conservatism in obstetrics. N Y Med J. |9|6;104:1-3.

9. Dauphinee JD. VBAC: Safety for the patient and the nurse. J Obstet Gynecol Neonatal Nurs. 2004;33:105-15.

I0. Socol ML. VBAC: is it worth the risk? Semin Perinatol. 2003;27:I05| I.

I I. Brill Y, Windrim R. Vaginal birth after Caesarean section: review of antenatal predictors of success. J Obstet Gynecol Can. 2003;25:275-86.

12. ACOG Committee on Obstetric Practice. Committee opinion. Induction of labor for vaginal birth after cesarean delivery. Obstet Gynecol. 2002;99:679-80.

13. Potter JE, Berquó E, Perpétuo IHO, Leal OF, Hopkins K, Souza MR, et al. Unwanted cesarean sections among public and private patients in Brazil: prospective study. Br Med J. 2001;323:1/55-8.

14. Cecatti JG, Andreucci CB, Cacheira PS, Besteti Pires HM, Pinto e Silva JLC, Aquino MMA. Fatores associados à realização de cesárea em primíparas com uma cesárea anterior. Rev Bras Ginecol Obstet. 2000;22:175-9.

15. Matias JP, Parpinelli MA, Cecatti JG, Passini Jr R. A prova de trabalho de parto aumenta a morbidade materna e neonatal em primíparas com uma cesárea anterior? Rev Bras Ginecol Obstet. 2003;25:255-60.

16. Brill Y, Kingdom J, Thomas J, Fraser W, Milne JK, Thomas M, et al. The management of VBAC at term: a survey of Canadian obstetricians. J Obstet Gynaecol Can. 2003;25:300-10.

17. Cecatti JG, Pires HM, Faundes A, Duarte Osis MJ. Factors associated with vaginal birth after previous cesarean section in Brazilian women. Rev Panam Salud Publica. 2005; 18:107-13.

18. Dodd JM, Crowther CA, Huertas E, Guise JM, Horey D. Planned elective repeat Caesarean section versus planned vaginal birth for women with a previous Caesarean birth (Cochrane Review). The Cochrane Library, Issue I, 2006. Oxford: Update Software.

19. Santos LC, Amorim MMR, Porto AMF, Azevedo EB, Mesquita CC. Fatores prognósticos para o parto transvaginal em pacientes com cesárea anterior. Rev Bras Ginecol Obstet. 1998;20:342-9.

20. Reddy UM, DiVito MM, Armstrong JC, Hyslop T, Wapner RJ. Population adjustment of the definition of the vaginal birth after cesarean rate. Am J Obstet Gynecol. 2000;183:1 166-9.

21. Pires HM, Cecatti JG, Faundes A. Factors associated with the trial of labor in primíparas women with one previous cesarean section. Rev Saúde Pública. 1999;33:342-8.

22. Socol ML, Peaceman AM. Vaginal birth after cesarean: an appraisal of fetal risk. Obstet Gynecol. 1999;93:674-9. 
23. O'Brien-Abel N. Uterine rupture during VBAC trial of labor: risk factors and fetal response. J Midwifery Womens Health. 2003;48:249-57.

24. Shipp TD, Zelop CM, Repke IT, Cohen A, Caughey AB, Lieberman E. Labor after previous cesarean: influence of prior indication and parity. Obstet Gynecol. 2000;95:913-6.

25. Linton A, Peterson MR, Williams TV. Effects of maternal characteristics on cesarean delivery rates among US Department of Defense healthcare beneficiaries, 1996-2002. Birth. 2004;31:3-11.

26. Lydon-Rochelle M, Holt VL, Easterling TR, Martin D. Risk of uterine rupture during labor among women with a prior cesarean delivery. $\mathrm{N}$ Engl | Med. 2001;345:3-8

27. Lieberman E. Risk factors for uterine rupture during a trial of labor after cesarean. Clin Obstet Gynecol. 2001;44:609-21.
28. Fisler RE, Cohen A, Ringer SA, Lieberman E. Neonatal outcome after trial of labor compared with elective repeat cesarean section. Birth. 2003;30:83-8

29. Mozurkewich EL, Hutton EK. Elective repeat cesarean delivery versus trial of labor: a meta-analysis of the literature from 1989 to 1999. Am J Obstet Gynecol. 2000;183:1187-97.

Artigo recebido: 14/02/06

Aceito para publicação: 31/01/07 